\title{
两个不同籽粒硬度小麦的比较蛋白组学分析
}

\author{
刘培勋 $^{1}$ 马小飞 ${ }^{2}$ 万洪深 ${ }^{1}$ 郑建敏 ${ }^{1}$ 罗江陶 ${ }^{1}$ 蒲宗君 ${ }^{1, *}$ \\ ${ }^{1}$ 四川省农业科学院作物研究所 / 农业农村部西南地区小麦生物学与遗传育种重点实验室, 四川成都 $610066 ;{ }^{2}$ 山西省农业科学院 \\ 小麦研究所, 山西临汾 041000
}

摘 要: 小麦是全球种植面积最大粮食作物，为全球 45 亿人提供日常蛋白和能量摄入的 $20 \%$ 。弄清小麦籽粒硬度遗传 基础, 对于改良小麦品质具有重大意义。为探讨不同硬度小麦种子的分子基础, 本实验选用西南麦区 2 个硬度差异极显著 的小麦品种川麦 66 和蜀麦 969 , 从蛋白水平上分析其种子蛋白差异表达情况, 利用 TMT 定量蛋白质组学技术(tandem mass $\operatorname{tags}$ )结合生物信息学分析，分析差异表达的蛋白及其功能和通路等富集情况。结果表明，鉴定并定量了有效蛋白 6020 个, 其中显著差异表达蛋白 113 个，在软质麦川麦 66 中上调表达的 69 个，下调表达的 44 个。差异蛋白 GO 富集分析共富集到 65 个 GO 条目, 达到极显著富集水平的包括生物过程的 1 个条目、细胞组成的 1 个条目和分子功能的 6 个条目。推测营 养库活性类蛋白、酶抑制剂活性类蛋白和谷胱甘肽代谢途径类蛋白可能参与小麦籽粒硬度形成。籽粒硬度相关蛋白可能 主要分布于细胞胞外区，具有防御作用。从系统发育分析推测, puroindolines 蛋白及其同源蛋白, 可能既作为小麦籽粒咜藏 蛋白, 同时还能作为酶抑制剂调控籽粒发育。本研究为进一步探索小麦籽粒硬度遗传机制提供了参考。

关键词: 小麦; 籽粒硬度; TMT; 蛋白质组

\section{Comparative proteomic analysis of two wheat genotypes with contrasting grain softness index}

\author{
LIU Pei-Xun ${ }^{1}$, MA Xiao-Fei ${ }^{2}$, WAN Hong-Shen ${ }^{1}$, ZHENG Jian-Min ${ }^{1}$, LUO Jiang-Tao ${ }^{1}$, and PU Zong-Jun ${ }^{1, *}$ \\ ${ }^{1}$ Crop Research Institute, Sichuan Academy of Agricultural Sciences / Key Laboratory of Wheat Biology and Genetic Improvement on Southwestern \\ China, Ministry of Agriculture and Rural Areas, Chengdu 610066, Sichuan, China; ${ }^{2}$ Wheat Research Institute, Shanxi Academy of Agricultural Sci- \\ ences, Linfen 041000, Shanxi, China
}

\begin{abstract}
Wheat is the crop most widely grown in the world and provides the daily protein and $20 \%$ food calories for 4.5 billion people. It is crucial to understand the genetic basis of grain hardness for improving wheat quality. In order to explore the molecular basis of the formation of wheat grain hardness, two wheat cultivars Chuanmai 66 and Shumai 969 with significant hardness difference in southwest wheat region were selected to analyze the proteins differential expression by TMT quantitative proteomics (tandem mass tags) and bioinformatic methods of function and pathway enrichment analysis. A total of 6020 effective proteins were identified and quantified, including 113 differentially expressed proteins (DEPs), of which 69 were up-regulated and 44 were down-regulated in soft wheat Chuanmai 66. These DEPs were enriched into 65 GO terms, including a biological process term, a cellular component term and six molecular function terms at extremely significant level. Based on the enrichment analysis, we suggested that nutrient reservoir activity proteins, enzyme inhibitor proteins and glutathione metabolism proteins might participate in the formation of wheat grain hardness, and grain hardness related proteins might mainly distribute in the extracellular region of cells and had defensive function. According to the phylogenetic analysis, it was inferred that puroindolines and its homologous proteins might be as not only wheat grain storage proteins, but also enzyme inhibitors regulating grain development. This study provides a basis for further exploring the genetic mechanism of wheat grain hardness.
\end{abstract}

Keywords: Triticum aestivum L.; grain hardness; TMT; protemics

本研究由国家自然科学基金项目(31671683, 31401383), 四川省财政创新能力提升工程(2016ZYPZ-016, 2019QNJJ-007, 2019QYXK034) 和四川省科技计划项目(2017JY0077)资助。

This study was supported by the National Natural Science Foundation of China $(31671683,31401383)$, the Financial Innovation Capacity Improvement Project of Sichuan Province (2016ZYPZ-016, 2019QNJJ-007, 2019QYXK034), and the Science and Technology Planning Project of Sichuan Province (2017JY0077).

*通信作者(Corresponding author): 蒲宗君, E-mail: pzjun68@163.com

第一作者联系方式: E-mail: littlefarmer@163.com; Tel: 028-84504231

Received (收稿日期): 2019-11-23; Accepted (接受日期): 2020-03-30; Published online (网络出版日期): 2020-04-26.

URL: http://kns.cnki.net/kcms/detail/11.1809.S.20200426.1402.006.html 
小麦硬度是评价小麦品质和影响小麦最终用途的重 要性状指标。硬质小麦适用于制作面包和面条等, 软质小 麦适用于制作饼干、糕点和酿酒等, 西南地区酿酒企业较 多, 酒曲以及酿酒过程对软质小麦的需求较大。弄清小麦 硬度遗传基础，对于改良小麦品质，培育满足不同需要的 小麦品种具有重大意义。

小麦籽粒硬度与蛋白质含量高度相关，硬质小麦蛋 白质含量比软质小麦高, 且在小麦籽粒中蛋白质和淀粉 以水溶性糖蛋白方式紧密结合。因此，籽粒硬度与蛋白质 含量、湿面筋含量呈显著正相关 ${ }^{[1]}$ 。硬质、高蛋白常被看 作是强筋小麦的标志, 而软质、低蛋白则被作为评价弱筋 小麦的依据 ${ }^{[2]}$ 。小麦种子蛋白主要由麦谷蛋白、醇溶蛋白、 球蛋白和清蛋白组成，其中谷蛋白和醇溶蛋白共同构成 种子贬藏蛋白。李硕碧等利用扫描电镜观察了小麦籽粒的 断面, 发现软质品种的淀粉近似球形, 清晰可见; 硬麦品 种籽粒的断面则很少见到完整淀粉粒，破碎淀粉粒较多， 胚乳结构相对致密 ${ }^{[3]}$ 。

普通小麦籽粒硬度由主效基因 puroindoline 控制 ${ }^{[4]}$, puroindoline 分为 Pina 和 Pinb 两种类型, 位于 $5 \mathrm{D}$ 染色体 短臂上, 二者高度同源且紧密连锁。软质对硬质为显性, 当 2 个基因同时存在时，小麦籽粒表现为软质，当 Pina 缺 失或 Pinb 发生变异时, 小麦籽粒表现为硬质, 能够解释 $60 \%$ 左右的变异 ${ }^{[5]}$ 。Pina 和 Pinb 均编码 148 个氨基酸序 列, 其氨基酸同源性约 $60 \%$, 分子量约为 $13 \mathrm{kD}$ 。研究发 现, 这种蛋白只存在于种子的胚乳细胞中, 而在其他组织 中没有 ${ }^{[6]}$ 。随着对 puroindoline 认识的逐步加深, 发现其 除了调节籽粒硬度之外, 还具有一定的抗病性 ${ }^{[7]}$ 。

与硬度相关的 gsp-1 (grain softness protein-1) 基因与 puroindoline 基因共同存在于 5DS 染色体上, 且高度同源, 但研究表明其对籽粒硬度影响不显著 ${ }^{[8]}$ 。Wilkinson 等 ${ }^{[9]}$ 在小麦 cDNA 中发现一种与 puroindoline $b$ 核苷酸序列相 似性高达 70\%的类 PIN 基因, 称为 puroindoline b-2, 对籽 粒硬度存在一定影响, Chen 等 ${ }^{[10]}$ 进一步发现该基因有多 种变异类型。随着基因组学的发展, 研究发现了一种新型 的败藏蛋白基因 avenin like $b$, 其所表达的蛋白与燕麦蛋 白相似, 为小麦品质的改良提供了参考 ${ }^{[11-12]}$ 。Furtadoet 等 ${ }^{[13]}$ 在小麦种子中发现一类新的基因 wheat bread making $(w b m)$, 对面筋质量、强度和延伸性有显著影响 ${ }^{[14]}$ 。对 puroindoline 同源蛋白家族分析发现, gsp-1、puroindolineb2、avenin like $\mathrm{b}$ 蛋白与 puroindoline 同属于 AAI_LTSS 超 级家族, 推测除 puroindoline 外, 存在其他基因, 尤其是 其同源基因, 也对籽粒硬度起作用 ${ }^{[15]}$ 。

目前, 对于小麦籽粒硬度的研究主要集中于 puroindoline 基因(Pina 和 Pinb), 但该基因尚不能完全解 释小麦籽粒硬度变异的机制 ${ }^{[16-17]}$ 。本研究利用 TMT (tandem mass tags)定量蛋白质组学技术, 结合小麦最新基 因组(iwgsc_refseqv1.0 2018)注释信息 ${ }^{[18]}$ 和 UniProt蛋白数 据库 ${ }^{[19]}$, 快速高通量地鉴定 2 个不同籽粒硬度小麦种子
中蛋白差异表达情况, 并通过生物信息学分析, 对差异表 达蛋白进行功能和通路等富集分析, 以期挖掘影响籽粒 硬度的潜在的新基因, 为小麦品质改良提供理论参考。

\section{1 材料与方法}

\section{1 试验设计}

四川主推小麦品种川麦 66 (系谱: 川麦 42/98-226// 01-3570) 是西南地区筋力最弱、硬度最低的品种之一 ${ }^{[20]}$, 蜀麦 969 (SHW-L1/SW8188//川育 18/3/川麦 42) 是四川主 推品种中筋力最强、硬度最高的品种之一 ${ }^{[21]}$, 两者都是川 麦 42 的衍生品种, 其亲缘关系相对较近。2018 年 10 月至 2019 年 5 月将其种植于四川省农业科学院德阳试验基地 $\left(31^{\circ} 14^{\prime} \mathrm{N}, 104^{\circ} 24^{\prime} \mathrm{E}\right), 3$ 个重复, 随机区组设计, 每个小区 行长 $1.5 \mathrm{~m}$, 行距 $0.3 \mathrm{~m}$, 共 10 行。播种时各施 $75 \mathrm{~kg} \mathrm{hm}^{-2}$ 的磷肥(过磷酸钙)和钾肥 (硫酸钾)作为基肥, 氮肥施尿素 $150 \mathrm{~kg} \mathrm{hm}^{-2}$, 分 2 次施用, 一半基施, 另一半作拔节肥。 收取成熟小麦籽粒(含水量 $12 \%$ ), 进行品质检测和蛋白表 达分析。使用单粒谷物测定仪 KSCS4100 检测小麦籽粒硬 度指数, 使用近红外谷物品质分析仪 Infratec 1241 检测粗 蛋白含量、湿面筋含量和淀粉含量。

\section{2 种子蛋白提取}

将冷冻的种子样品低温研磨成粉, 加入蛋白裂解液 $\left(100 \mathrm{mmol} \mathrm{L}^{-1}\right.$ 碳酸氢铵、6 $\mathrm{mol} \mathrm{L}^{-1}$ 尿素、0.2\%SDS, pH 8.0) 充分裂解, 然后在 $4^{\circ} \mathrm{C}$ 下 $12,000 \times g$ 离心 $15 \mathrm{~min}$, 取上清 液, 加入终浓度 $10 \mathrm{mmol} \mathrm{L}^{-1}$ DTTred 于 $56^{\circ} \mathrm{C} 反$ 应 $1 \mathrm{~h}$, 再 加入足量 IAM, 于室温避光反应 $1 \mathrm{~h}$ 。加入 4 倍体积的 $-20^{\circ} \mathrm{C}$ 预冷丙酮, 于 $-20^{\circ} \mathrm{C}$ 条件下沉淀 $2 \mathrm{~h}$, 最后在 $4^{\circ} \mathrm{C}$ 下 $12,000 \times g$ 离心 $15 \mathrm{~min}$, 收集沉淀。经重悬清洗后, 加入适 量蛋白溶解液 $\left(6 \mathrm{~mol} \mathrm{~L}^{-1}\right.$ 尿素、 $\left.100 \mathrm{mmol} \mathrm{L}^{-1} \mathrm{TEAB}, \mathrm{pH} 8.5\right)$ 溶解蛋白沉淀。使用 Bradford 蛋白质定量试剂盒, 绘制标 准曲线，计算待测样品的蛋白浓度。

\subsection{TMT 标记和液质检测}

2 个品种, 3 次重复, 共 6 个蛋白样品经酶切与除盐 ${ }^{[22]}$ 后, 分别加入 $0.1 \mathrm{~mol} \mathrm{~L}^{-1} \mathrm{TEAB}$ 缓冲液和乙腈溶解的含不 同同位素标签的 TMT (Tandem Mass Tags)标记 ${ }^{[23]}$ 试剂, 室温下颠倒混匀反应 $2 \mathrm{~h}$ 。加入终浓度 $8 \%$ 氨水终止反应, 取等体积标记后的样品混合，除盐后冻干。使用 L-3000HPLC 系统进行馏分分离, 每分钟收集 1 管, 合并 为 10 个馏分, 冻干后各加入 $0.1 \%$ 甲酸溶解。使用 EASY-nLCTM1200 纳升级 UHPLC 系统，进行液相色谱检 测。使用 QExactiveTMHF-X 质谱仪, NanosprayFlex ESI) 离子源, 数据依赖型采集模式进行质谱检测, 设一级质谱 分辨率为 $60,000\left(200 \mathrm{~m} \mathrm{z}^{-1}\right)$ 。选取全扫描中离子强度 TOP40 的母离子使用高能碰撞裂解(HCD)方法碎裂, 进行 二级质谱检测，设二级质谱分辨率为 $15,000\left(200 \mathrm{~m} \mathrm{z}^{-1}\right)$, 最终生成质谱检测原始数据(.raw)。

1.4 蛋白鉴定

合并 UniProt 数据库 ${ }^{[19]}$ 和最新小麦基因组 ${ }^{[18]}$ 注释文 
件 IWGSC RefSeq v1.0 annotation (154,254 sequences)作为 最终的搜索数据库。利用 Proteome Discoverer 2.2 软件对 检索结果进一步过滤: 可信度在 $99 \%$ 以上的谱肽为可信 PSMs (peptide spectrum matches), 至少包含一个 unique 肽 段(特有肽段)的蛋白为可信蛋白, 只保留可信的谱肽和蛋 白, 通过 FDR 验证，去除掉 FDR 大于 $1 \%$ 的肽段和蛋白。 对鉴定到的蛋白进行蛋白注释, 包括 GO 注释 ${ }^{[24]}$ 、 KEGG 注释 ${ }^{[25]} 、 \mathrm{COG}$ 注释 ${ }^{[26]}$ 和结构域注释(IPR) ${ }^{[27]}$ 。

\section{5 蛋白定量分析和差异表达蛋白功能分析}

根据原始下机的谱图峰面积可以得到各个样品中每 个 PSM 的相对定量值, 后再根据鉴定出的 Unique 肽段中 所包含所有 PSM 的定量信息校正得到 Unique 肽段的相对 定量值, 最后根据每个蛋白质包含的所有 Unique 肽段的 定量信息校正得到每个蛋白的相对定量值。将每个蛋白在 比较样品对中的所有生物重复定量值的均值的比值作为 差异倍数(Fold Change, FC)。将每个蛋白在样品中的相对 定量值进行 $T$-test 检验, $P$ 值作为显著性指标。当 $\mathrm{FC} \geq 1.5$, 且 $P \leq 0.05$ 时, 蛋白表现为表达量上调, 当 $\mathrm{FC} \leq 0.67$, 同 时 $P \leq 0.05$ 时, 蛋白表现为表达量下调。针对篮选出来的 差异蛋白进行 GO、KEGG 功能富集分析, 与所有鉴定到 的蛋白背景相比, 应用超几何检验计算 $P$ 值, 以 $P \leq 0.05$ 为阈值, 满足此条件的条目 (或路径)定义为在差异蛋白质 中显著富集的条目(或路径)。

1.6 差异表达蛋白与 puroindolines 蛋白系统发育分析

前人对于小麦籽粒硬度的研究主要集中于 puroindoline 基因(Pina 和 Pinb), 该蛋白主要在软质麦中存在和表达。从 UniProt 数据库下载小麦 Pina 和 Pinb 蛋白。将本研究中鉴定 出的在软质麦川麦 66 中较硬质麦蜀麦 969 中显著上调表达 的蛋白与 puroindolines 蛋白一起, 利用 Clustal X 2.0 进行序 列比对, 利用 MEGA 7.0 软件邻接法构建系统发育树, 分析 差异表达蛋白与 puroindolines 蛋白的同源关系 ${ }^{[28]}$ 。

\section{2 结果与分析}

\subsection{2 个小麦籽粒样品质分析}

经检测, 川麦 66 的平均籽粒硬度指数为 25.2 , 蜀麦 969 的平均籽粒硬度指数为 40.2 。籽粒粗蛋白含量、湿面 筋含量和淀粉含量检测主要结果见图 1 , 川麦 66 的籽粒 硬度指数、粗蛋白含量和湿面筋含量均低于蜀麦 969 , 淀 粉含量则高于蜀麦 969 , 经 $t$ 检验, 差异均达到显著水平。

\section{2 蛋白鉴定}

通过数据库搜索, 鉴定到二级谱图(total spectra)数量 为 85,497 个, 肽段 (Peptide) 数为 32,552 个, 蛋白数 (protein)为 6061 个。利用 $\mathrm{GO} 、 \mathrm{KEGG}$ 和 $\mathrm{COG}$ 数据库对 鉴定到的蛋白质进行功能注释, 利用 Pfam、ProDom、 SMART 等结构域的数据库进行结构域注释(IPR), 利用模 式结构或特征进行功能未知蛋白的结构域注释, 共注释 到 6020 个蛋白, 注释结果统计见图 2, 有 2819 个蛋白可 同时被 4 种数据库注释。

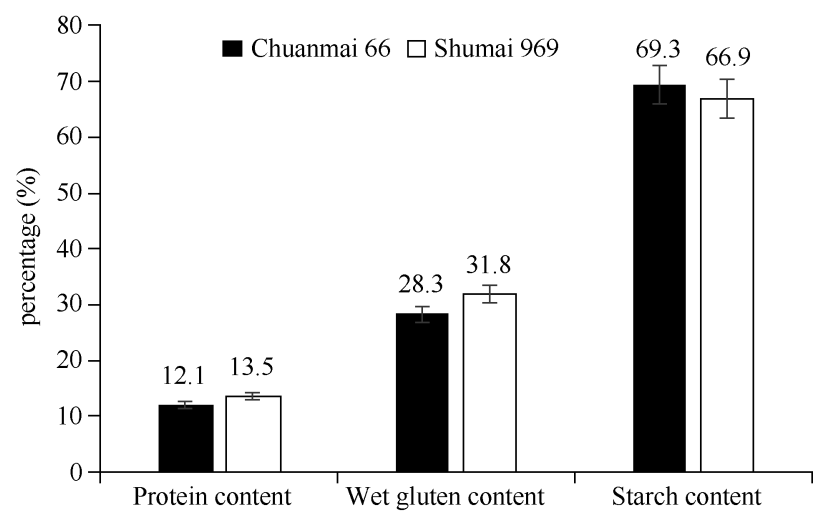

图 1 川麦 66 和蜀麦 969 籽粒主要品质指标比较

Fig. 1 Comparison of the grain quality between Chuanmai 66 and Shumai 969

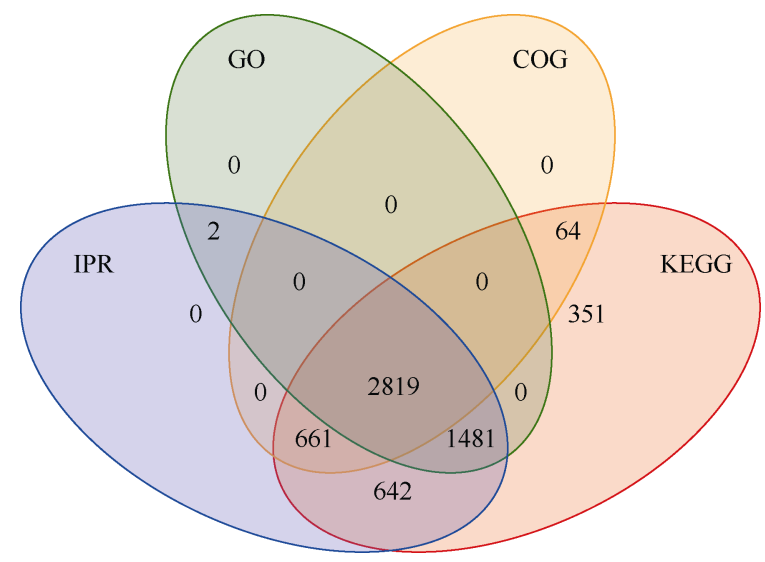

图 2 蛋白质功能注释结果

Fig. 2 Result of protein functional annotations

\section{3 差异表达蛋白}

根据限制条件篮选的差异表达蛋白(DEP), 在注释到 的 6020 个蛋白中, 对每个蛋白质差异倍数以 2 为底取对 数, 将 $P$ 值以 10 为底取对数的绝对值, 做出火山图, 观察 不同蛋白在不同样品间比较时的上调、下调情况, 结果见 图 3。2 个样品差异表达蛋白(DEP)总数为 113 , 其中软质 麦川麦 66 中较硬质麦蜀麦 969 中显著上调的蛋白为 69 个, 显著下调的蛋白为 44 个。

\section{4 差异蛋白 GO 富集分析}

$\mathrm{GO}$ 显著性富集分析能分析差异蛋白行使的主要生物 学功能。2 个小麦样品中, 表达差异显著的 113 个蛋白经 $\mathrm{GO}$ 富集分析, 富集到 65 个 $\mathrm{GO}$ 条目中, 去除仅包含 1 个 差异蛋白的条目, 剩余 33 个可靠 $\mathrm{GO}$ 条目。其中, 达到显 著富集 $(P<0.05)$ 水平的 $\mathrm{GO}$ 条目为 10 个，属于生物过程 1 个, 细胞组分 1 个, 分子功能的 8 个(图 4)。达到极显著富 集 $(P<0.01)$ 水平的条目为 8 个, 生物过程条目有 1 个, 为 防御反应过程(defense response); 细胞组分条目有 1 个, 为胞外区(extracellular region); 分子功能条目有 6 个, 包 括营养库活性(nutrient reservoir activity)、酶抑制剂活性 (enzyme inhibitor activity)、酶调节活性(enzyme regulator 


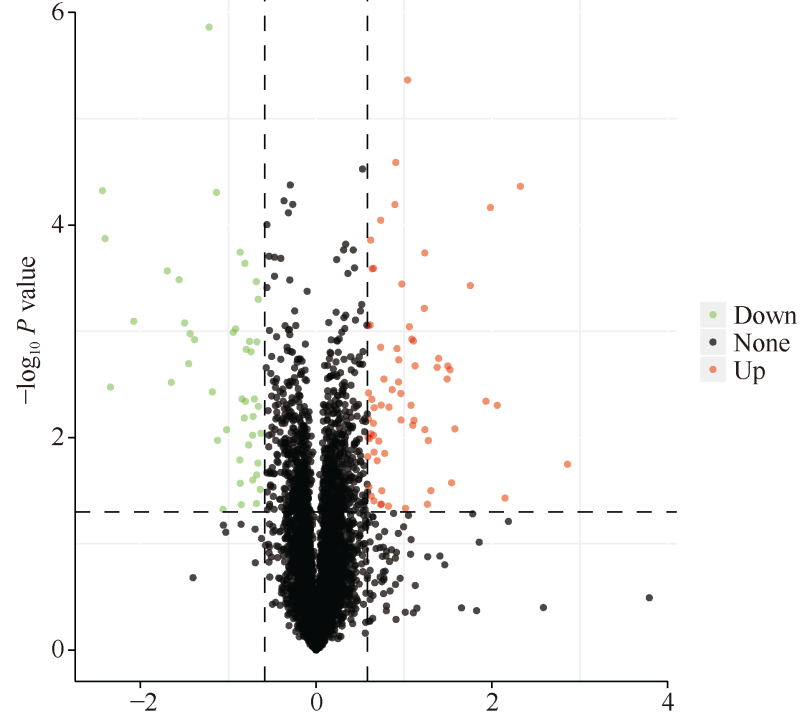

图 3 差异表达蛋白火山图

Fig. 3 Differential protein volcano map

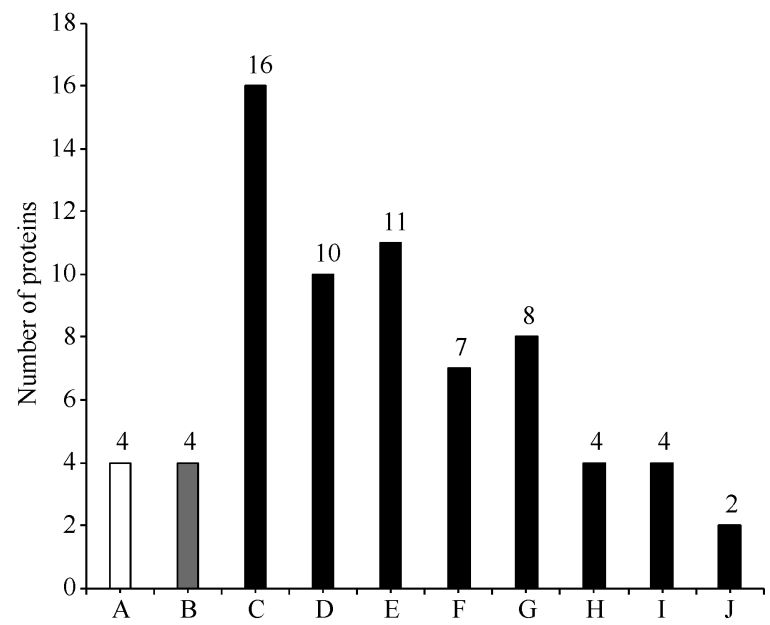

图 4 GO 富集柱状图

Fig. 4 Differential protein GO enrichment results

A 属于生物过程(BP), B 属于细胞组分 $(\mathrm{CC}), \mathrm{C} 、 \mathrm{D} 、 \mathrm{E} 、 \mathrm{~F} 、 \mathrm{G} 、 \mathrm{H} 、$ $\mathrm{I} 、 \mathrm{~J}$ 属于分子功能(MF)。A：防御反应; B: 胞外区; C: 营养库活 性; D：酶抑制剂活性；E：酶调节活性；F：丝氨酸型内肽酶抑制 剂活性; G: 肽链内切酶抑制剂活性; H: 天冬氨酸型内肽酶活性; I: 电子载体活性; J: 几丁质结合。

A belongs to BP (biological process), B belongs to $\mathrm{CC}$ (cellular component), C, D, E, F, G, H, I, J belong to MF (molecular function). A: defense response; B: extracellular region; C: nutrient reservoir activity; D: enzyme inhibitor activity; E: enzyme regulator activity; F: serine-type endopeptidase inhibitor activity; G: endopeptidase inhibitor activity; H: aspartic-type endopeptidase activity; I: electron carrier activity; J: chitin binding.

activity)、丝氨酸型内肽酶抑制剂活性 (serine-type endopeptidase inhibitor activity)、肽链内切酶抑制剂活性 (endopeptidase inhibitor activity)和天冬氨酸型内肽酶活性 类(aspartic-type endopeptidase activity)。

达到极显著富集水平的分子功能类的 6 个 $\mathrm{GO}$ 条目, 有 1 个为营养库活性, 其余 5 个条目均涉及酶抑制剂活 性。营养库活性条目中包含 16 个差异表达蛋白(表 1), 其 中在川麦 66 中显著上调表达的有 7 个, 下调表达的 9 个。 该类别中包含的蛋白主要有低分子谷蛋白亚基、燕麦相似 蛋白、 $\alpha$ 和 $\gamma$-醇溶蛋白及萌发素类蛋白。 5 个涉及酶抑制 剂活性的条目中, 多个蛋白均同时属于不同的 GO 条目。 经整理，属于酶抑制剂活性类的差异表达蛋白共 15 个, 包括 $\alpha$-淀粉酶、胰蛋白酶、转化酶、糜蛋白酶等(表 2)。 其中, 10 个蛋白在川麦 66 中显著上调表达, 5 个下调表达, puroindoline b 蛋白富集于该类别中，在川麦 66 中较蜀麦 969 差异表达倍数达到 2.8 倍。

\section{5 差异蛋白 KEGG 富集分析}

通过 KEGG Pathway 显著性富集分析，可了解差异蛋 白参与的最主要生化代谢途径和信号转导途径。通过 KEGG 富集, 113 个差异蛋白中有 48 个差异蛋白富集到 26 个生化代谢途径，达到显著富集的有谷胱甘肽代谢 (Glutathione metabolism)、光合作用-天线蛋白(phtosynthesisantenna proteins)和类胡夢卜素代谢(carotenoid biosynthesis)。 但光合作用-天线蛋白代谢和类胡夢卜素代谢分别仅有 1 个差异蛋白, 可靠性较差。谷胱甘肽代谢条目达到极显著 富集水平, 包含 6 个差异表达蛋白, 其中 4 个上调表达, 2 个下调表达(表 3)。

2.6 上调表达蛋白与 puroindolines 蛋白同源关系分析

将提取出的 69 个在软质麦川麦 66 中显著上调表达的 蛋白与从 UniProt 数据库下载的小麦 puroindolines 蛋白 (puroindoline a 和 puroindoline b) 共同构建系统发育树 (图 5)。对属于营养库活性类别、酶抑制剂活性类别和谷 胱甘肽代谢途径的蛋白分别用空心正方形、三角形和圆形 标记。可见，7 个营养库活性类蛋白在进化树中，与 puroindolines 蛋白聚到一起; 10 个酶抑制剂活性类蛋白中 有 4 个与 puroindolines 蛋白聚到一起; 谷胱甘肽代谢类蛋 白则没有与 puroindolines 蛋白聚到一起。

\section{3 讨论}

本研究中所用 2 个硬度差异较大的品种, 都是来自西 南麦区, 并且是同一个亲本的衍生品种, 其亲缘关系较近, 一定程度上可以排除不同麦区品种之间对习性、抗性的差 异以及部分遗传背景的差异。通过定量蛋白质组学 TMT 标记结合液相色谱质谱联用技术和生物信息学分析, 共 鉴定出 6020 个蛋白，在 2 个不同籽粒硬度小麦种子中表 达差异显著的蛋白 113 个, 69 个蛋白在川麦 66 中高表达, 44 个蛋白在蜀麦 969 中高表达。鉴定的蛋白数量和差异 表达蛋白数量均较高, 证明 TMT 技术是一种高通量高分 辨率的蛋白定量检测技术 ${ }^{[29]}$ 。 
表 1 营养库活性类相关差异表达蛋白(川麦 66 vs. 蜀麦 969)

Table 1 DEPs with nutrient reservoir activity (Chuanmai 66 vs. Shumai 969)

\begin{tabular}{|c|c|c|c|}
\hline $\begin{array}{l}\text { 蛋白名称 } \\
\text { Protein ID }\end{array}$ & $\begin{array}{c}\text { 描述 } \\
\text { Description }\end{array}$ & $\begin{array}{c}\text { 差异倍数 } \\
\text { Fold change }\end{array}$ & $\begin{array}{l}\text { 上调/下调 } \\
\text { Up/down }\end{array}$ \\
\hline B2Y2Q6 & B2Y2Q6_WHEAT LMW-B2 & 1.5425 & Up \\
\hline B2BZC7 & B2BZC7_WHEAT LMW-m glutenin subunit 0154A5-M & 1.8737 & Up \\
\hline A0A173DQZ1 & A0A173DQZ1_WHEAT type-b avenin-like protein & 3.8163 & Up \\
\hline Q8H0J5 & Q8H0J5_WHEAT low molecular weight glutenin subunit (fragment) & 5.0056 & Up \\
\hline TraesCS7A01G035300.1 & Gliadin-like avenin & 2.9853 & Up \\
\hline Q6WZC3 & Q6WZC3_WHEAT low molecular weight glutenin subunit & 2.1622 & Up \\
\hline TraesCS4A01G453400.1 & Gamma-gliadin & 7.2606 & Up \\
\hline A0A286QTK1 & A0A286QTK1_WHEAT avenin-like protein A2 & 0.5984 & Down \\
\hline F8SGL3 & F8SGL3_WHEAT low-molecular-weight glutenin subunit & 0.5493 & Down \\
\hline Q0QBR3 & Q0QBR3_WHEAT LMW-glutenin P3-5 & 0.3190 & Down \\
\hline $\mathrm{B} 2 \mathrm{Y} 2 \mathrm{R} 3$ & B2Y2R3_WHEAT low molecular weight glutenin subunit & 0.5725 & Down \\
\hline B6UKN9 & B6UKN9_WHEAT gamma-gliadin & 0.6340 & Down \\
\hline TraesCS2A01G211800.1 & Germin-like protein 1-1 & 0.5875 & Down \\
\hline A0A2P1H6A2 & A0A2P1H6A2_WHEAT alpha-gliadin & 0.5908 & Down \\
\hline A0A0K2QJY6 & A0A0K2QJY6_WHEAT alpha/beta-gliadin & 0.6081 & Down \\
\hline I0IT65 & I0IT65_WHEAT alpha-gliadin & 0.6241 & Down \\
\hline
\end{tabular}

表 2 酶抑制剂类相关差异表达蛋白(川麦 66 vs. 蜀麦 969)

Table 2 DEPs with enzyme inhibitor activity (Chuanmai 66 vs. Shumai 969)

\begin{tabular}{|c|c|c|c|}
\hline $\begin{array}{l}\text { 蛋白名称 } \\
\text { Protein ID }\end{array}$ & $\begin{array}{c}\text { 描述 } \\
\text { Description }\end{array}$ & $\begin{array}{c}\text { 差异倍数 } \\
\text { Fold change }\end{array}$ & $\begin{array}{l}\text { 上调/下调 } \\
\mathrm{Up} / \text { down }\end{array}$ \\
\hline Q5UHH6 & Q5UHH6_WHEAT 0.19 dimeric alpha-amylase inhibitor (fragment) & 1.6788 & Up \\
\hline TraesCS5D01G004300.1 & Puroindoline-b, protease inhibitor/seed storage/LTP family & 2.8227 & Up \\
\hline P81713 & IBB3_WHEAT Bowman-Birk type trypsin inhibitor & 1.5092 & Up \\
\hline TraesCS4A01G460900.1 & Invertase inhibitor, plant invertase/pectin methylesterase inhibitor & 1.5169 & Up \\
\hline TraesCS1D01G028500.1 & Chymotrypsin inhibitor & 2.3465 & Up \\
\hline TraesCS5D01G561800.1 & Invertase inhibitor, plant invertase/pectin methylesterase inhibitor & 2.0582 & Up \\
\hline A0A080YTU1 & A0A080YTU1_WHEAT uncharacterized protein & 1.5779 & Up \\
\hline A0A2X0S1F0 & A0A2X0S1F0_WHEAT peptidase A1 domain-containing protein & 1.9149 & Up \\
\hline TraesCS4D01G205800.1 & ADP-ribosylation factor GTPase-activating protein & 1.6650 & Up \\
\hline TraesCS7A01G502500.1 & Eukaryotic aspartyl protease family protein & 2.3542 & Up \\
\hline TraesCS3D01G467500.1 & Eukaryotic aspartyl protease family protein & 0.3700 & Down \\
\hline TraesCS4D01G250000.1 & Dimeric alpha-amylase inhibitor & 0.3659 & Down \\
\hline TraesCS1D01G265900.1 & Wound-induced protease inhibitor & 0.5474 & Down \\
\hline TraesCS3D01G025700.1 & Trypsin inhibitor & 0.1974 & Down \\
\hline TraesCS6B01G407700.1 & Aspartic proteinase nepenthesin-1 & 0.6473 & Down \\
\hline
\end{tabular}


表 3 谷胱甘肽代谢途径相关差异表达蛋白(川麦 66 vs. 蜀麦 969)

Table 3 DEPs related to Glutathione metabolism (Chuanmai 66 vs. Shumai 969)

\begin{tabular}{clcc}
\hline 蛋白名称 & \multicolumn{1}{c}{$\begin{array}{c}\text { 上调/下调 } \\
\text { Protein ID }\end{array}$} & \multicolumn{1}{c}{$\begin{array}{c}\text { 差异倍数 } \\
\text { Fold change }\end{array}$} & Up/down \\
\hline TraesCS3D01G491400.1 & Description & 2.5968 & Up \\
TraesCS2B01G096200.1 & Ascorbate peroxidase & 1.5695 & Up \\
TraesCS3A01G488200.1 & Glutathione S-transferase & 1.5215 & $\mathrm{Up}$ \\
TraesCS3B01G536100.1 & Glutathione S-transferase & 1.9499 & $\mathrm{Up}$ \\
TraesCS3D01G445400.1 & Glutathione S-transferase & 0.6058 & Down \\
W5D4D9 & W5D4D9_WHEAT uncharacterized protein & 0.5675 & Down \\
\hline
\end{tabular}

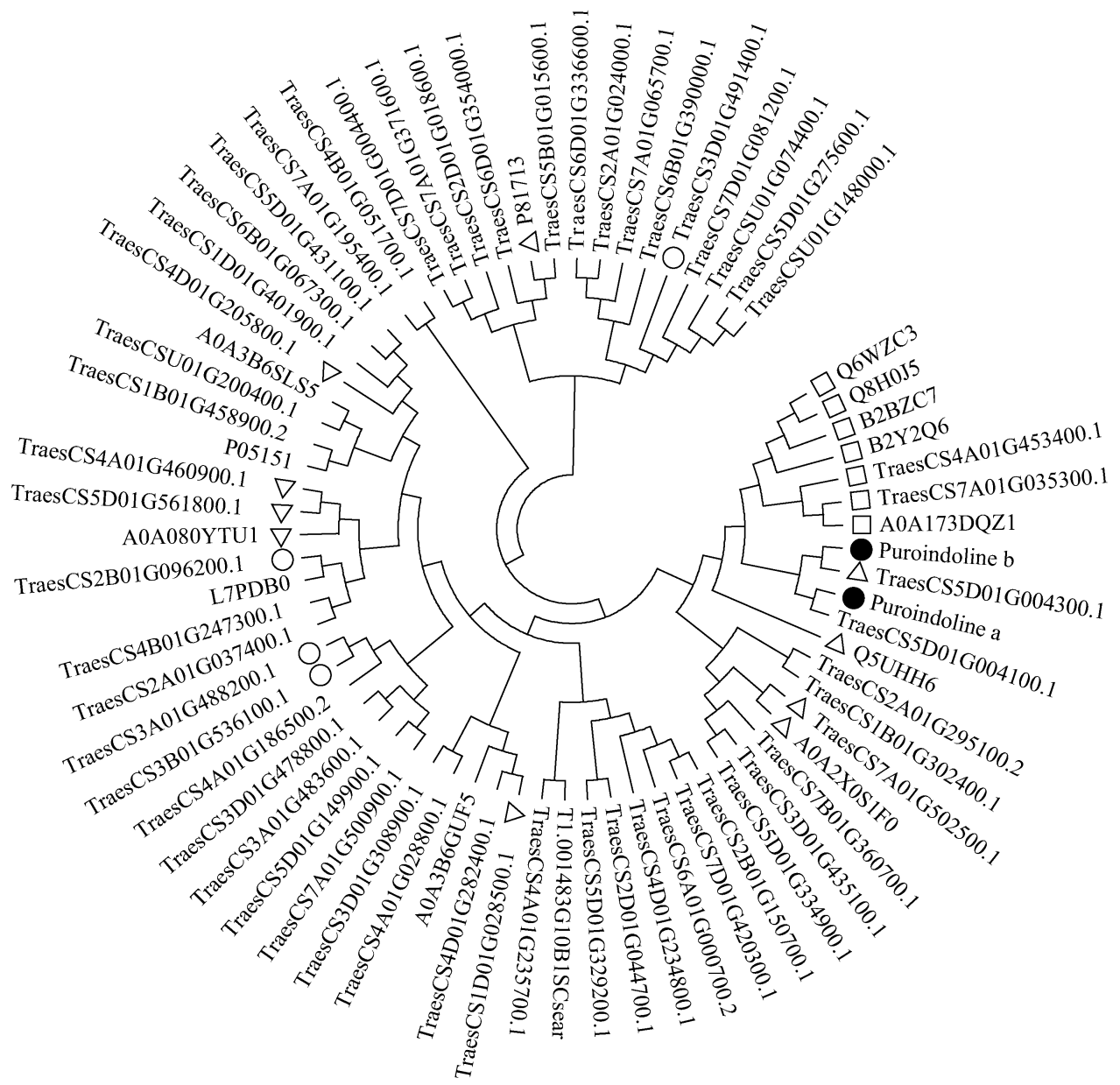

图 5 软质麦中上调表达蛋白与 puroindolines 蛋白共同构建的系统发育树

Fig. 5 Phylogenetic tree constructed by differentially expressed proteins and the puroindolines

带实心圆形的为 puroindolines 蛋白; 带空心正方形的为营养库活性类别; 带空心三角形的为酶抑制剂活性类别; 带空心圆形的为谷胱 甘肽代谢途径蛋白。

The proteins with solid circles are puroindolines, the proteins with squares belong to nutrient reservoir activity category, the proteins with triangles belong to enzyme inhibitor activity category, and the proteins with circles belong to glutathione metabolism category.

通过 GO 富集方法, 2 个样品中表达差异显著的蛋白, 极显著富集到生物过程、细胞组成和分子功能类别的数量 分别为 $1 、 1$ 和 6 个。 1 个生物过程条目为防御反应类别, 1 个细胞组分条目为胞外区类别。推测小麦籽粒硬度相关蛋 白与小麦抗逆反应有关, 可能主要分布于细胞胞外区。 Kim 等研究表明, 影响小麦籽粒硬度最重要的蛋白

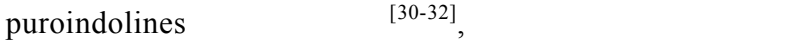
蛋白参与抗逆作用可互为支撑。6 个分子功能类 $\mathrm{GO}$ 条目 中, $P$ 值最小的条目为营养库活性类别, 另外 5 个均与酶 抑制剂活性相关。

营养库活性类别, 主要是低分子谷蛋白和醇溶蛋白 等贮藏蛋白，是小麦种子的重要结构组成成分。贮藏蛋白 
的种类和表达量, 决定着小麦种子中蛋白的含量和组成, 从而影响小麦硬度。酶抑制剂类蛋白对硬粒的影响目前未 见专门报道。以 puroindolines 蛋白为例, 该蛋白具有 $\alpha$ 淀粉酶抑制剂功能，假设 puroindolines 高表达可有效抑制 $\alpha$-淀粉酶, 从而防止种子中淀粉水解。由此, 可解释软质 小麦中淀粉粒较完整、近似球形，而硬质小麦淀粉粒破坏 严重、胚乳结构致密 ${ }^{[3]}$ 。硬质麦中, 蛋白质和淀粉以水溶 性糖蛋白方式紧密结合 ${ }^{[1]}$, 可能与缺少淀粉酶抑制剂, 造 成淀粉表面水解成糖有关。不管是营养库类别还是酶抑制 剂类别, 均有部分蛋白相对表达量与籽粒柔软度呈正相 关, 另一部分则为负相关, 其作用机制有待进一步研究。

通过 KEGG Pathway 显著性富集分析，富集到 26 个 生化代谢途径，其中只有 3 个途径达到显著富集水平，其 中谷胱甘肽代谢途径达到极显著富集水平, 推测相关蛋 白对籽粒硬度有一定的作用。TraesCS3A01G 488200.1、 TraesCS3B01G536100.1 和 TraesCS3D01G 445400.1 蛋白 描述均为谷胱甘肽转化酶, 且分别位于 $3 \mathrm{~A} 、 3 \mathrm{~B}$ 和 $3 \mathrm{D}$ 染 色体，应该是分布于 3 号染色体群的同源基因。但之前未 见相关报道, 其影响机制有待进一步研究。

Puroindolines 基因(Pina 和Pinb)目前被认为是影响小 麦籽粒硬度的最主要基因, 但尚不能完全解释小麦籽粒 硬度变异机制 ${ }^{[16,33]}$ 。经序列比对和查阅小麦基因组汶览器 (JBrowse $^{[34]}$,TraesCS5D01G004100.1 和 TraesCS5D01G 004300.1 分别就是 Pina 和 Pinb 蛋白, 该实验中 puroindolines 蛋白在软质麦中显著高表达, 与前人研究结 果一致 ${ }^{[35]}$ 。将 puroindolines 蛋白与川麦 66 中显著上调表 达的蛋白共同构建进化树, 7 个营养库活性类蛋白和 4 个 酶抑制剂蛋白与 puroindolines 蛋白聚为一类。可见, 从蛋 白序列同源性角度，营养库活性类蛋白和部分酶抑制剂 类蛋白与 puroindolines 蛋白有着高度同源性, 可视为同一 蛋白家族成员, 因此与 puroindolines 蛋白具有相似的分子 功能。某些小麦籽粒䛎藏蛋白, 可能兼具酶抑制剂功能。

\section{References}

[1] Bakhella M, Hoseney R C, Lookhart G L. Hardness of Moroccan wheats. Cereal Chem, 1990, 67: 246-250.

[2] 吴宏亚, 蒋正宁, 王玲, 程顺和. 小麦籽粒硬度及其对面粉加 工品质影响的研究进展. 江苏农业学报, 2014, 30: 437-441.

Wu H Y, Jiang Z N, Wang L, Cheng S H. Research progress in wheat kernel hardness and its effect on milling quality. Jiangsu $J$ Agric Sci, 2014, 30: 437-441 (in Chinese with English abstract).

[3] 李硕碧. 小麦籽粒胚乳结构性状的研究. 西北农林科技大学 学报(自然科学版). 2002, 30(5): 7-10.

Li S B. Study on structural characters of grain endosperm of wheat. $J$ Northwest A\&F Univ (Nat Sci Edn), 2002, 30(5): 7-10 (in Chinese with English abstract).

[4] Giroux M J, Morris C F. Wheat grain hardness results from highly conserved mutations in the friabilin components puroindoline a and b. Proc Nat Acad Sci USA, 1998, 95: 6262-6266.

[5] Mods C F. Puroindolines: the molecular genetic basis of wheat grain hardness. Plant Mol Biol, 2002, 48: 633-647.

[6] Wiley P R, Tosi P, Evrard A, Lovegrove A, Jones H D, Shewry P R. Promoter analysis and immunolocalisation show that puroindoline genes are exclusively expressed in starchy endosperm cells of wheat grain. Plant Mol Biol, 2007, 64: 125-136.

[7] 陈锋, 董中东, 程西永, 詹克慧, 许海霞, 崔党群. 小麦 puroindoline 及其相关基因分子遗传基础研究进展. 中国农业 科学, 2010, 43: 1108-1116.

Chen F, Dong Z D, Cheng X Y, Zhan K H, Xu H X, Cui D Q. Advances in research of molecular genetics of puroindoline and its related genes in wheat. Sci Agric Sin, 2010, 43: 1108-1116 (in Chinese with English abstract)

[8] Gollan P, Smith K, Bhave M. Gsp-1 genes comprise a multigene family in wheat that exhibits a unique combination of sequence diversity yet conservation. J Cereal Sci, 2007, 45: 184-198.

[9] Wilkinson M, Wan Y, Tosi P, Leverington M, Snape J, Mitchell R A C, Shewry P R. Identification and genetic mapping of variant forms of puroindoline $\mathrm{b}$ expressed in developing wheat grain. $J$ Cereal Sci, 2008, 48: 722-728.

[10] Chen F, Beecher B S, Morris C F. Physical mapping and a new variant of puroindoline $b-2$ genes in wheat. Theor Appl Genet, 2010, 120: 745-751.

[11] Anderson O D, Hsia C C, Adalsteins A E, Lew E L, Kasarda D D. Identification of several new classes of low-molecular-weight wheat gliadin-related proteins and genes. Theor Appl Genet, 2001, 103: 307-315.

[12] Zhang Y, Hu X, Islam S, She M, Peng Y, Yu Z, Zhang J. New insights into the evolution of wheat avenin-like proteins in wild emmer wheat (Triticum dicoccoides). Proc Natl Acad Sci USA, 2018, 115: 13312-13317.

[13] Furtado A, Bundock P C, Banks P M, Fox G, Yin X, Henry R J. A novel highly differentially expressed gene in wheat endosperm associated with bread quality. Sci Rep, 2015, 5:10446.

[14] Guzmán C, Xiao Y, Crossa J, González-Santoyo H, Huerta J, Singh R, Dreisigacker S. Sources of the highly expressed wheat bread making (wbm) gene in CIMMYT spring wheat germplasm and its effect on processing and bread-making quality. Euphytica, 2016, 209: 689-692.

[15] 刘培勋, 万洪深, 郑建敏, 罗江陶, 蒲宗君. 小麦 PIN 基因家族 的鉴定及表达分析. 中国农业科学, 2020, 53(12): 2321-2330.

Liu P X, Wan H S, Zheng J M, Luo J T, Pu Z J. Genome-wide identification and expression analysis of PIN genes family in wheat. Sci Agric Sin, 2020, 53(12): 2321-2330 (in Chinese with English abstract).

[16] Ayala M, Guzmán C, Peña R J, Alvarez J B. Genetic diversity and molecular characterization of puroindoline genes (Pina-D1 and $P i n b-D 1$ ) in bread wheat landraces from Andalusia (Southern Spain). J Cereal Sci, 2016, 71: 61-65.

[17] Chen M, Wilkinson M, Tosi P, He G, Shewry P. Novel puroindoline and grain softness protein alleles in Aegilops species with the C, D, S, M and U genomes. Theor Appl Genet, 2005, 111: 1159-1166.

[18] Alaux M, Rogers J, Letellier T, Flores R, Alfama F, Pommier C, Guerche C. Linking the international wheat genome sequencing consortium bread wheat reference genome sequence to wheat genetic and phenomic data. Genome Biol, 2018, 19: 111. 
[19] UniProt: the universal protein knowledgebase. Nucleic Acids Res, 2016, 45: D158-D169.

[20] 郑建敏, 蒲宗君. 白皮优质弱筋小麦新品种川麦 66. 四川农 业科技, 2015, (2): 24-24.

Zheng J M, Pu Z J. Chuanmai 66, a new white wheat variety with high quality and soft grain. Sci Technol Sichuan Agric, 2015, (2): 24-24 (in Chinese).

[21] 蒋进, 王淑荣, 张连全, 刘利, 冯晓, 左娟. 四川省 “十二五” 期间育成小麦新品种的品质分析. 种子, 2017, 36(2): 95-99.

Jiang J, Wang S R, Zhang L Q, Liu L, Feng X, Zuo J. Quality analysis of wheat varieties released in Sichuan province during the 12th Five-Year Plan. Seed, 2017, 36(2): 95-99 (in Chinese).

[22] Wu J, An Y, Pu H, Shan Y, Ren X, An M, Ji J. Enrichment of serum low-molecular-weight proteins using $\mathrm{C} 18$ absorbent under urea/dithiothreitol denatured environment. Anal Biochem, 2010, 398: 34-44.

[23] Thompson A, Schäfer J, Kuhn K, Kienle S, Schwarz J, Schmidt G, Hamon C. Tandem mass tags: a novel quantification strategy for comparative analysis of complex protein mixtures by MS/MS. Anal Chem, 2003, 75: 1895-1904.

[24] Ashburner M, Ball C A, Blake J A, Botstein D, Butler H, Cherry J M, Harris M A. Gene ontology: tool for the unification of biology. Nat Genet, 2000, 25: 25-29.

[25] Kanehisa M, Goto S, Hattori M, Aoki-Kinoshita K F, Itoh M, Kawashima S, Hirakawa M. From genomics to chemical genomics: new developments in KEGG. Nucl Acids Res, 2006, 34: 354-357.

[26] Tatusov R L, Fedorova N D, Jackson J D, Jacobs A R, Kiryutin B, Koonin E V, Rao B S. The COG database: an updated version includes eukaryotes. BMC Bioinform, 2003, 4: 41.

[27] Finn R D, Attwood T K, Babbitt P C, Bateman A, Bork P, Bridge A J, Gough J. InterPro in 2017: beyond protein family and domain annotations. Nucleic Acids Res, Jan 2017; doi: 10.1093/ nar/gkw1107.

[28] Kumar S, Stecher G, Tamura K. MEGA7: Molecular evolutionary genetics analysis version 7.0 for bigger datasets. Mol Biol Evol, 2016, 33: 1870 .

[29] 宋奇琦, Pratiksha SINGH, Rajesh Kumar SINGH, 宋修鹏, 李 海碧, 农友业, 杨丽涛, 李杨瑞. 基于 iTRAQ技术的甘蔗受黑 穗病菌侵染蛋白组分析. 作物学报, 2019, 45: 55-69.

Song Q Q, Pratiksha S, Rajesh K S, Song X P, Li H B, Nong Y Y, Yang L T, Li Y R. Proteomic analysis of sugarcane-Sporisorium scitamineum interaction based on iTRAQ technique. Acta Agron Sin, 2019, 45: 55-69.

[30] Kim K H, Feiz L, Dyer A T, Grey W, Hogg A C, Martin J M, Giroux M J. Increased resistance to Penicillium seed rot in transgenic wheat over-expressing puroindolines. J Phytopathol, 2012, 160: 243-247.

[31] Dhatwalia V K, Sati O P, Tripathi M K, Kumar A. Puroindoline: antimicrobial wheat endosperm specific protein. J Agric Technol, 2011, 7: 903-906.

[32] Phillips R L, Palombo E A, Panozzo J F, Bhave M. Puroindolines, Pin alleles, hordoindolines and grain softness proteins are sources of bactericidal and fungicidal peptides. J Cereal Sci, 2011, 53: 112-117.

[33] Nirmal R C, Furtado A, Wrigley C, Henry R J. Influence of gene expression on hardness in wheat. PLoS One, 2016, 11: e0164746.

[34] Skinner M E, Uzilov A V, Stein L D, Mungall C J, Holmes I H. Jbrowse: a next-generation genome browser. Genome Res, 19: 1630-1638.

[35] Li Y, Mao X, Wang Q, Zhang J, Li X, Ma F, Sun F, Chang J, Chen M, Wang Y, Li K, Yang G X, He G. Overexpression of puroindoline agene in transgenic durum wheat (Triticum turgidum ssp. durum) leads to a medium-hard kernel texture. Mol Breed, 2014, 33: 545-554. 